\title{
CONTAGEM DE EOSINÓFILOS PERIFÉRICOS NA REJEIÇÃO AGUDA DO ENXERTO HEPÁTICO
}

\author{
Countin of Blood eosinophils in acute cellular rejection of hepatic graft \\ Olival Cirilo Lucena da Fonseca Neto', Ludmila Rodrigues Oliveira Costa², Rebeca Mangabeira Correia², \\ Caio Rodrigo de Oliveira Melo², George Felipe Bezerra Darce'2, Paulo Ricardo Bispo Siqueira², \\ Priscylla Jennie Monteiro Rabelo², Paulo Sérgio Viera de Melo², Américo Gusmão Amorim², \\ Claudio Moura Lacerda ${ }^{1}$
}

\section{RESUMO}

Introdução: A rejeição aguda pós-transplante de fígado é uma das complicações mais comuns de disfunção do enxerto hepático. O padrão-ouro para identificação dessa condição é a biópsia, que, contudo, é um procedimento invasivo. Dessa maneira, a literatura vem estudando marcadores não invasivos para prever a rejeição aguda; dentre esses, destaca-se a eosinofilia periférica. Objetivo: Avaliar a contagem de eosinófilos periféricos durante os episódios de rejeição ao enxerto hepático, correlacionando com os níveis de transaminases hepáticas. Métodos: Trata-se de um estudo observacional retrospectivo, com pareamento entre as variáveis estudadas: taxas de sensibilidade, especificidade, valor preditivo e acurácia. Resultados: Rejeição ao transplante foi detectada em 29,33\% do total de pacientes. Dentre os rejeitados, o valor de aspartato aminotransferase teve aumento em $65,90 \%$ dos casos, sensibilidade diagnóstica de $59,18 \%$, especificidade de $81,13 \%$, valor preditivo positivo de $65,91 \%$, valor preditivo negativo de $85,15 \%$ e acurácia de $76,67 \%$. A alanina aminotransferase aumentou em $34,09 \%$, com sensibilidade diagnóstica de $60,00 \%$, especificidade de $90,57 \%$, valor preditivo positivo de $34,09 \%$, valor preditivo negativo de $76,80 \%$ e acurácia de $74,00 \%$. A contagem relativa de eosinófilos teve aumento em $29,54 \%$ dos casos, sensibilidade de $22,03 \%$, especificidade $56,60 \%$, valor preditivo positivo de $29,55 \%$, valor preditivo negativo de $65,93 \%$ e acurácia de $48,67 \%$. Conclusão: É certo que o presente trabalho e a literatura não mostram resultados estaticamente favoráveis e convergentes à utilização da eosinofilia periférica juntamente com as transaminases para prever rejeição aguda hepática. Ainda assim, a baixa sensibilidade apontada nas estatísticas não descarta a utilização da eosinofilia periférica como auxílio diagnóstico.

Descritores: Transplante de Fígado; Eosinófilos; Rejeição do Enxerto; Biomarcadores

\section{Instituição:}

1 Serviço de Cirurgia Geral e Transplante Hepático - Hospital Universitário Oswaldo Cruz, Recife, PE, Brasil

2 Faculdade de Ciências Médicas de Pernambuco, UPE, Recife, PE, Brasil

\section{Correspondência:}

Olival Cirilo Lucena Da Fonseca Neto

Rua Jacobina, 45, Apt. 1002 - CEP 52011-180, Recife-PE.

Tel.:(81) 99989-0208

Email: olivalneto@globo.com

Recebido em: 24/01/2020

Aceito em: 27/02/2020

\section{INTRODUÇÃO}

A rejeição aguda pós-transplante hepático é uma das complicações mais comuns encontradas no período de pós-operatório precoce; ocorrendo em até quatro semanas. ${ }^{1}$ Embora isso possa ser causa de disfunção do enxerto, pacientes são, geralmente, assintomáticos e as transaminases hepáticas - aspartato aminotransferase (AST) e alanina aminotransferase (ALT) - sozinhas não são suficientemente sensíveis para o diagnóstico da condição. $^{2}$

O padrão-ouro para a identificação da rejeição celular aguda é a biópsia, a qual demonstra um infiltrado 
linfocítico periportal, que se estende ao parênquima do fígado, assim como a invasão de células inflamatórias no endotélio vascular e dano do ducto biliar. ${ }^{3}$ Contudo, a biópsia pode gerar complicações ao paciente e, nem sempre, a mesma permite a diferenciação entre rejeição aguda e outras lesões. ${ }^{4}$

Há uma discussão na literatura acerca da rejeição aguda clínica, isto é, o diagnóstico dessa condição através de marcadores de disfunção do enxerto e do quadro clínico do paciente, sem a utilização da biópsia. ${ }^{2}$ Todavia, não é um conceito bem estabelecido, visto que ainda não há resultados totalmente satisfatórios sobre marcadores simples de medir, com baixo custo e suficientemente sensíveis e específicos. ${ }^{1}$

A eosinofilia periférica vem sendo enfatizada como atuante nos mecanismos fisiopatológicos da rejeição celular aguda em transplante de órgãos sólidos e sugerida como uma ferramenta de auxílio diagnóstica nas últimas duas décadas. As pesquisas acerca desse marcador são bastante controversas, e alguns estudos apontam dados estatísticos não tão promissores. ${ }^{5-11}$ Diante disso, o presente trabalho busca avaliar a contagem de eosinófilos periféricos durante os episódios de rejeição ao enxerto hepático, correlacionando-os com os níveis de transaminases hepáticas.

\section{MÉTODOS}

Trata-se de um estudo observacional retrospectivo. A partir do pareamento entre as variáveis estudadas, foi possível avaliar a correlação entre a contagem de eosinófilos e a progressão de rejeição ao enxerto, sem, entretanto, intervir diretamente na relação analisada. A população de estudo foi de pacientes submetidos a transplante de fígado no Serviço de Cirurgia Geral e Transplante Hepático do Hospital Universitário Oswaldo Cruz (HUOC), Recife/PE.

A coleta de dados foi feita por meio de acesso aos exames contidos nos prontuários dos pacientes submetidos a transplante de fígado. O Teste Exato de Fisher foi utilizado visando comprovar se as ocorrências nas categorias definidas são ou não equivalentes nas populações estudadas, sendo um teste comparativo em que é calculada, diretamente, a significância, de forma direta, sem recorrência à estatística intermediária. O intervalo de confiança é definido como $95 \%$ e a margem de erro aceita é definida como $5 \%$; dessa forma, o valor de $P$ é considerado significante se $<0.05$, extremamente significante se $<0.01$, e não significante se $>0.05$.

A análise dos dados deu-se por meio da elaboração de planilhas eletrônicas desenvolvidas no Microsoft Excel, versão 365. Calcularam-se as taxas de sensibilidade, especificidade, valor preditivo, acurácia, e, também, estabeleceu-se uma comparação entre os valores da contagem de eosinófilos e de transaminases. Definiu-se como paciente com diagnóstico de rejeição aguda aqueles nos quais observamos os níveis de transaminases elevarem-se no pós-operatório precoce, e, em seguida, reduzirem-se após o aumento da imunossupressão.

O desenvolvimento da pesquisa implicou na observância da resolução que norteia a pesquisa envolvendo seres humanos, portanto sendo enviado ao Comitê de Ética em Pesquisa em Seres Humanos da UNIDADE DE PESQUISA CLÍNICA DO HUOC/PROCAPE (CAAE=0122.0.106.000-11). Comprometemo-nos a garantia da privacidade das informações obtidas, a partir dessa pesquisa.

\section{RESULTADOS}

\section{Dados demográficos}

Foram avaliados 151 prontuários de pacientes de transplante hepático realizados entre agosto de 1999 e agosto de 2008, dentre os quais, 95 eram do sexo masculino e 56 do sexo feminino, com idade média de $48,16 \pm 21,89$ anos, apresentando como resultado do teste do qui-quadrado o valor de 14,8157 e $p=0,0051$.

\section{Etiologia das doenças hepáticas}

As indicações de transplante prevalentes foram enquadradas no diagnóstico sindrômico de Cirrose NãoColestática $(59,49 \%)$, seguida de Cirrose Colestática e Neoplasias, ambas com $11,39 \%$. Os principais diagnósticos etiológicos foram cirrose por hepatite C $(15,54 \%)$, cirrose por hepatite alcoólica $(10,81 \%)$ e cirrose criptogênica $(6,75 \%)$ (Figura 1).

Figura 1: Diagnóstico etiológico dos transplantados hepáticos no período de agosto de 1999 a agosto de 2008
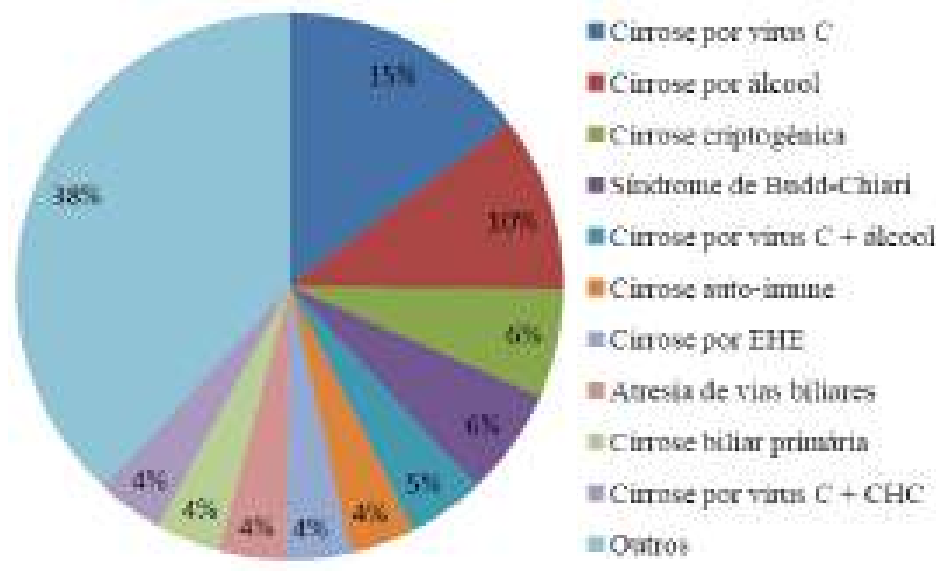
Olival Cirilo Lucena da Fonseca Neto, Ludmila Rodrigues Oliveira Costa, Rebeca Mangabeira Correia, Caio Rodrigo de Oliveira Melo, George Felipe Bezerra Darce, Paulo Ricardo Bispo Siqueira, Priscylla Jennie Monteiro Rabelo, Paulo Sérgio Viera de Melo, Américo Gusmão Amorim, Claudio Moura Lacerda

\section{Incidência da Rejeição aguda}

Rejeição ao transplante foi detectada em $29,33 \%$ do total de pacientes, sendo a sua distribuição apresentada por faixa etária na figura 2 . O teste do qui-quadrado teve resultados de 0,8095 e 1,9337 para a rejeição separada por sexo e faixa etária, respectivamente. O p foi de 0,3683 e 0,7480 para as mesmas condições.

Figura 2: Distribuição da idade dos pacientes sem rejeição aguda (Não) e com rejeição aguda (Sim), pós transplante hepático

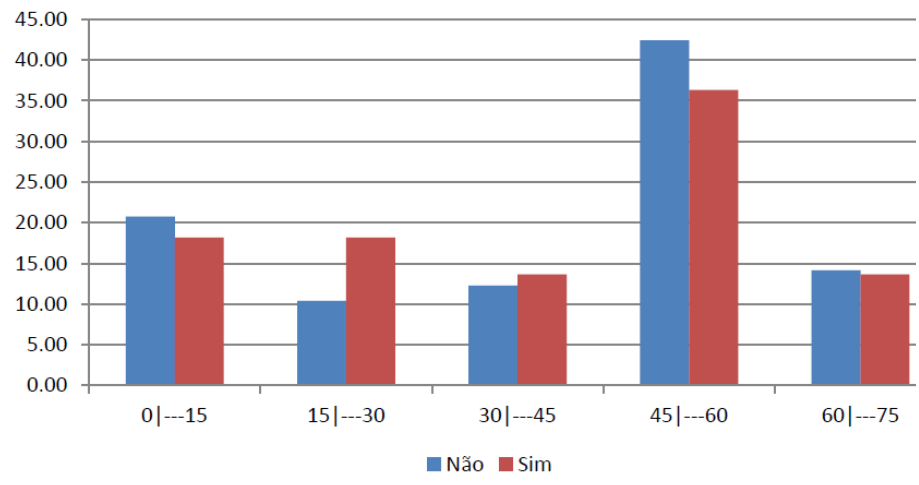

\section{Marcadores diagnósticos da rejeição aguda}

AST apresentou aumento em $65,90 \%$ dos casos de rejeição aguda, sendo usado como ponto de corte o valor de 60 . O mesmo mostrou sensibilidade $59,18 \%$ para o diagnóstico da condição, especificidade de $81,13 \%$, valor preditivo positivo de $65,91 \%$, valor preditivo negativo de $85,15 \%$ e acurácia de $76,67 \%$.

ALT apresentou aumento em $34,09 \%$ dos casos de rejeição aguda, sendo usado como ponte de corte o valor de 300. A mesma mostrou sensibilidade de $60,00 \%$ para o diagnóstico da condição, especificidade de $90,57 \%$, valor preditivo positivo de $34,09 \%$, valor preditivo negativo de $76,80 \%$ e acurácia de $74,00 \%$.

A contagem relativa de eosinófilos apresentou aumento em $29,54 \%$ dos casos, com $4 \%$ como ponto de corte. A mesma mostrou sensibilidade de $22,03 \%$, especificidade $56,60 \%$, valor preditivo positivo de $29,55 \%$, valor preditivo negativo de $65,93 \%$ e acurácia de $48,67 \%$.

Curva de crescimento dos marcadores diagnósticos no pós-operatório

A curva de AST nos pacientes com rejeição aguda teve um perfil de aumento a partir do oitavo dia de pósoperatório, enquanto que, naqueles sem rejeição, houve queda gradual no pós-operatório (Figura 3).

Em relação à $A L T$, o aumento iniciou-se, na maioria dos casos, a partir do sétimo dia de pós-operatório e com perfil crescente, até o décimo segundo dia. Nos pacientes sem rejeição, foram obtidos resultados com perfil de queda da transaminase (Figura 4).
Figura 3: Contagem de aspartato aminotransferase (AST) em pacientes sem rejeição aguda (Não) e em pacientes com rejeição aguda (Sim) nos 12 primeiros dias pósTransplante Hepático.

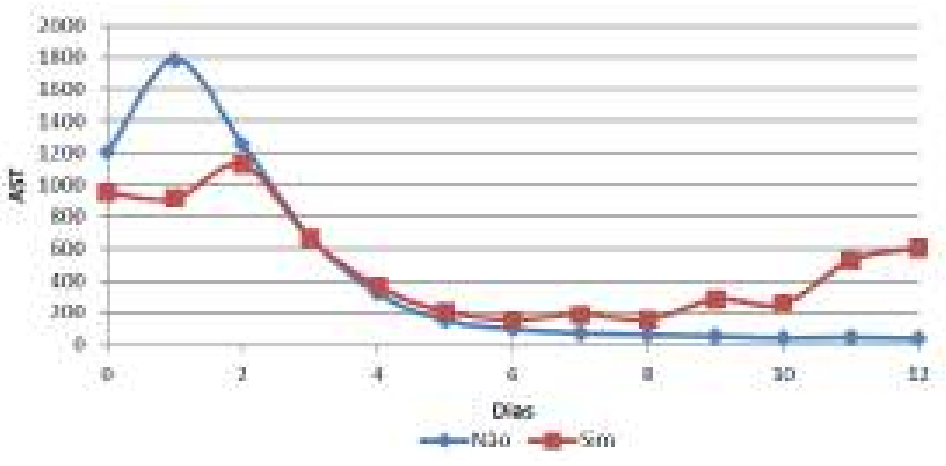

Figura 4: Contagem de alanina aminotransferase (ALT) em pacientes sem rejeição aguda (Não) e em pacientes com rejeição aguda (Sim) nos 12 primeiros dias pósTransplante Hepático

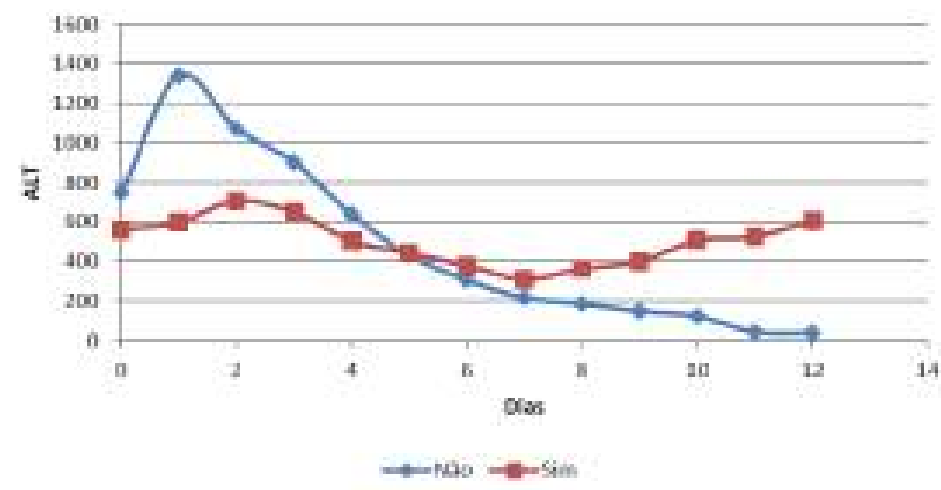

Quanto à contagem relativa de eosinófilos periféricos nos pacientes com rejeição aguda, a média dos valores aumentou acima de $4 \%$ a partir do quinto dia de pósoperatório e decresceu a partir do décimo dia após o transplante (Figura 5).

Figura 5: Contagem relativa de eosinófilos periféricos em pacientes com rejeição aguda (Sim) e em pacientes sem rejeição aguda (Não) nos 12 primeiros dias pósTransplante Hepático

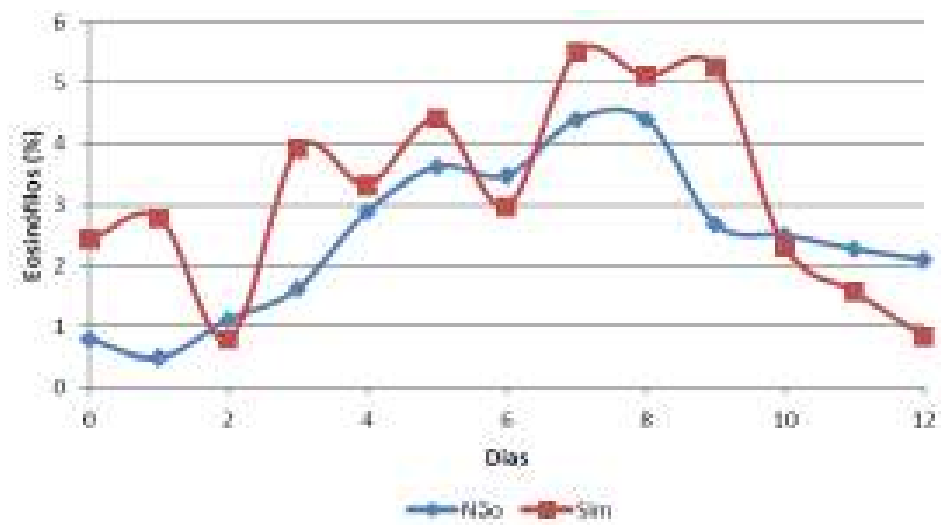




\section{DISCUSSÃO}

Diversos estudos apontam a eosinofilia sanguínea como preditor de rejeição aguda pós-transplante hepático. ${ }^{7}$ Apesar do mecanismo dessa correlação não ser bem entendido, a identificação desse marcador contribui para uma decisão racional de biopsiar o aloenxerto e/ou tratá-lo empiricamente. ${ }^{2}$

No presente estudo, a eosinofilia periférica apresentou menor sensibilidade diagnóstica para rejeição ajuda do que os marcadores ALT e AST, assim como menor especificidade. Apesar da baixa sensibilidade da eosinofilia sanguínea, estudos mostram que esse marcador apresenta maior valor preditivo para casos de rejeição moderada a grave, sendo assim, pouco sensível para identificação de pacientes com rejeição leve. ${ }^{2,5}$ Uma possível justificativa é a redução de eosinófilos periféricos pela corticoterapia pósoperatória, a qual mascara principalmente os casos leves de rejeição. ${ }^{12,13}$

Em contrapartida à baixa sensibilidade, a especificidade da eosinofilia periférica para prever rejeição aguda é consideravelmente maior, destacando-se a raridade de situações que podem acarretar em aumento desse marcador em pacientes transplantados. A literatura aponta poucas condições as quais podem elevar a contagem de eosinófilos sanguíneos em pacientes que não apresentam rejeição aguda: infecção por parasita, malignidade como linfoma e alergia medicamentosa. 5 A infecção por parasita é um ponto importante, principalmente no público transplantado brasileiro, o qual é mais humilde socioeconomicamente.

No presente estudo, não houve comparação entre eosinófilos sanguíneos e do enxerto, visto a precaução de nosso Serviço em realizar biópsia apenas em pacientes que apresentem um quadro clínico sugestivo de rejeição aguda. Dessa forma, não foi possível fazer uma associação estatística entre os dois parâmetros. Apesar disso, é de extrema valia buscar um padrão entre eosinofilia do enxerto e periférica, como forma de prever os pacientes que necessitam da biópsia para confirmação do diagnóstico de rejeição. Foster PF et al ${ }^{7}$ mostraram que, nos casos em que a eosinofilia do enxerto não acompanhou a eosinofilia sanguínea, apenas uma entre 30 biópsias apontaram rejeição ajuda. Isto é, muitos casos do aumento de eosinófilos sanguíneo podem ocorrer, sem ser, necessariamente, devido à rejeição aguda. Apesar do resultado pouco favorável de Foster PF et al, ${ }^{7}$ Kishi $\mathrm{Y}$ et al ${ }^{8}$ apontaram que a eosinofilia periférica, contabilizada três dias antes da realização da biópsia, previu a rejeição aguda com especificidade de $94 \%$.
É certo que os resultados dos estudos são controversos e questionam a importância da eosinofilia periférica como ferramenta diagnóstica, todavia é preciso atentar-se à real necessidade da realização da biópsia. Um estudo retrospectivo observou que pacientes com rejeição subclínica, isto é, com alterações da biópsia, porém sem manifestação clínica de disfunção hepática, quando tratados com doses maior de corticoide, apresentaram taxa de sobrevivência de enxerto menor que os pacientes com rejeição subclínica sem tratamento, devido às complicações por infecção do tratamento. Isso gera um questionamento da necessidade de uma rotina precoce de biópsia em pacientes sem quadro clínico sugestivo de rejeição ajuda, visto que a má interpretação das alterações histopatológicas pode induzir ao aumento da imunossupressão do paciente, corroborando as complicações para o mesmo. ${ }^{14}$

Nossos dados mostraram que as transaminases ALT e AST apresentaram alta especificidade e uma curva de crescimento nos pacientes com rejeição aguda diferente dos sem rejeição (Figuras 3 e 4). Há uma discussão difusa na literatura acerca da importância das transaminases para o diagnóstico da rejeição aguda. Matsukara A et al ${ }^{15}$ verificaram que todos os pacientes que apresentaram rejeição aguda, também apontaram eosinofilia periférica e aumentos das transaminases hepáticas, em até dois dias após o diagnóstico da rejeição com biópsia. Em contrapartida, há estudos que não demonstram diferença significante das transaminases hepáticas entre o grupo com rejeição aguda e o grupo controle, ${ }^{5,16}$ sendo observados até $48,2 \%$ dos pacientes com episódios de rejeição com enzimas hepáticas inalteradas. Uma limitação de nosso estudo é que não foi realizado um coorte entre pacientes com rejeição e sem rejeição, dessa forma não podemos sugerir se houve diferença desses marcadores entre os grupos apontados.

Outro ponto importante é o acompanhamento da contagem de eosinófilo para prever a melhora do paciente após o tratamento da rejeição. Uma publicação mostrou que, de 45 pacientes com rejeição aguda comprovada, após tratamento com corticoide, não houve diferença significante da contagem de eosinófilo sanguíneo entre os que melhoraram, os que permaneceram estáveis e os que deterioraram da condição. ${ }^{5}$ Observa-se que os eosinófilos sanguíneos caem drasticamente, independentes da resposta ao tratamento. ${ }^{8}$ Por outro lado, uma segunda publicação apontou uma associação significativa entre a melhora da eosinofilia do enxerto e a redução de contagem de eosinófilos sanguíneos. (WANG et al., 2013) Uma possível justificativa da divergência de resultados é a eosinopenia causada pelo corticoide, ${ }^{12,13}$ o que dificulta um padrão de correlação entre a redução de contagem de eosinófilos periféricos e a resposta ao tratamento da rejeição. 
Olival Cirilo Lucena da Fonseca Neto, Ludmila Rodrigues Oliveira Costa, Rebeca Mangabeira Correia, Caio Rodrigo de Oliveira Melo, George Felipe Bezerra Darce, Paulo Ricardo Bispo Siqueira, Priscylla Jennie Monteiro Rabelo, Paulo Sérgio Viera de Melo, Américo Gusmão Amorim, Claudio Moura Lacerda

Nossos dados mostram a necessidade de protocolos que analisem não só a eosinofilia sanguínea e aumento das transaminases hepáticas, mas também, outros parâmetros além da biópsia hepática, para guiar uma decisão terapêutica diante da rejeição aguda.

\section{CONCLUSÃO}

É certo que o presente trabalho e a literatura não mostram resultados estaticamente favoráveis e convergentes à utilização da eosinofilia periférica, juntamente com as transaminases, para prever rejeição aguda hepática e acompanhamento da evolução do enxerto. Todavia, em um contexto em que nem sempre a biópsia hepática é possível, devido aos riscos trazidos aos pacientes e a capacidade do Serviço de Transplante, a baixa sensibilidade apontada nas estatísticas não descarta a utilização da eosinofilia periférica aliada às transaminases hepáticas como auxílio diagnóstico para a rejeição aguda pós-transplante hepático.

\section{ABSTRACT}

Introduction: Acute rejection after liver transplantation is one of most common complications of the liver graft. The gold-standard to identify this condition is the biopsy. However, it is an invasive procedure that can can pose serious complications to the patient. The literature has studied non-invasive biomarkers that could predict acute rejection and peripheral eosionophils is one of them. Purpose: to assess peripheral eosinophils and liver transaminases during acute rejection of liver graft at Hospital Universitário Oswaldo Cruz. Methods: It is an observational study assessing the following variables: sensitivity rates, specificity, predictive value, and accuracy. Results: Acute rejection was detected in $29.33 \%$ of patients. In patients with acute rejection, the AST counting were high in $65.90 \%$, with $59.18 \%$ of diagnostic sensibility, $81.13 \%$ of diagnostic specificity, positive predictive value of $65.91 \%$, negative predictive value of $85.15 \%$, and $76.67 \%$ accuracy; the ALT counting were high in $34.09 \%$, with $60.00 \%$ of diagnostic sensibility, $90.57 \%$ of diagnostic specificity, $34.09 \%$ of positive predictive value, $76.80 \%$ of negative predictive value and $74,00 \%$ of accuracy; peripheral eosinophils were high in $29,54 \%$, with $22,03 \%$ of diagnostic sensibility, $56,60 \%$ of diagnostic specificity, $29.55 \%$ of positive predictive value, negative predictive value of $65.93 \%$ and accuracy of $48.67 \%$. Conclusion: The present study, as well as the literature suggest the limited role of eosinophilia as a predictor for acute rejection. However, we agree that the peripheral eosinophils can be used as a diagnostic tool.

Keywords: Liver Transplantation; Eosinophils; Graft Rejection; Biomarkers.

\section{REFERÊNCIAS}

1. Wang YC, Wu TJ, Wu TH, Lee CF et al. The risk factors to predict acute rejection in liver transplantation. Transplant Proc. 2012 Mar;44(2):526-8.

2. Rodriguez-Peralvarez M, Germani G, Tsochatzis E, Rolando N, Luong TV, Dhillon AP, et al. Predicting severity and clinical course of acute rejection after liver transplantation using blood eosinophil count. Transpl Int. 2012 May;25(5):555-63.

3. Dousset B, Hubscher SG, Padbury RT, Gunson BK, Buckels JA, Mayer AD, et al. Acute liver allograft rejection-is treatment always necessary? Transplantation. 1993 Mar;55(3):529-34.
4. Tisone G, Orlando G, Vennarecci G, Palmieri GP, Pisani F, Strati E, et al. Spontaneous resolution of severe acute rejection in liver transplantation. Transplant Proc. 1999 Dec;31(8):3164-6.

5. Barnes EJ, Abdel-Rehim MM, Goulis Y, Abou Ragab M, Davies S, Dhillon A. Applications and limitations of blood eosinophilia for the diagnosis of acute cellular rejection in liver transplantation. Am J Transplant. 2003 Apr;3(4):432-8.

6. Florman S, Schiano T, Kim L, Maman D, Levay A, Gondolesi $\mathrm{G}$, et al. The incidence and significance of late acute cellular rejection (> 1000 days) after liver transplantation. Clin Transplant. 2004 Apr;18(2):152-5 
7. Foster PF, Sankary HN, Hart M, Ashmann M, Williams JW. Blood and graft eosinophilia as predictors of rejection in human liver transplantation. Transplantation. 1989 Jan;47(1):72-4.

8. Kishi Y, Sugawara Y, Tamura S, Kaneko J, Akamatsu N, Togashi J, et al. Is blood eosinophilia an effective predictor of acute rejection in living donor liver transplantation? Transpl Int. 2005 Oct;18(10):1147-51.

9. Nagral A, Quaglia A, Sabin CA, Dhillon AP, Bearcroft CP, Millar A, et al. Blood and graft eosinophils in acute cellular rejection of liver allografts. Transplantation Proceedings. 2001;33(4):2588-93.

10.Sanada Y, Ushijima K, Mizuta K, Urahashi T, Ihara Y, Wakiya T, et al. Prediction of Acute Cellular Rejection by Peripheral Blood Eosinophilia in Pediatric Living Donor Liver Transplantation. Transplant Proc. 2012 Jun;44(5):1341-5.

11.Wang G-Y, Li H, Liu W, Zhang J, Zhu H-B, Wang G-S, et al. Elevated Blood Eosinophil Count Is a Valuable Biomarker for Predicting Late Acute Cellular Rejection After Liver Transplantation. Transplant Proc. 2013 Apr;45(3):1198-200.
12.Andersen V, Bro $\square$ Rasmussen F, Hougaard K. Autoradiographic Studies of Eosinophil Kinetics: Effects of Cortisol*. Cell Proliferation. 1969;2(2):139-46.

13.Meagher LC, Cousin JM, Seckl JR, Haslett C. Opposing effects of glucocorticoids on the rate of apoptosis in neutrophilic and eosinophil granulocytes. Journal of Immunology. 1996 Jun 1;156(11):4422-8.

14.Tippner C, Nashan B, Hoshino K, Schmidt-Sandte E, Akimaru $\mathrm{K}$, Boker KHW, et al. Clinical and subclinical acute rejection early after liver transplantation - Contributing factors and relevance for the long-term course. Transplantation. 2001 Sep 27;72(6):1122-8.

15. Matsukura A, Kita Y, Harihara Y, Tanaka H, Ito M, Kawarasaki H.Does peripheral blood eosinophilia predict allograft rejection in living-related liver transplantation?Transplant Proc. 1999 Nov;31(7):2729-30.

16.Morgul MH, Splith K, Leonhardt C, Raschzok N, ReutzelSelke A, Schmuck RB et al. The value of microparticles in detecting acute rejection episodes after liver transplantation. Biomarkers. 2018Feb;23(1):25-32. 